\title{
Generalized Quadratic Linearization of Machine Models
}

\author{
Parvathy Ayalur Krishnamoorthy, ${ }^{1}$ Kamaraj Vijayarajan, ${ }^{2}$ and Devanathan Rajagopalan ${ }^{1}$ \\ ${ }^{1}$ Department of Electrical and Electronics Engineering Department, Hindustan of Science \& Technology, Chennai 603103, India \\ ${ }^{2}$ Department of Electrical and Electronics Engineering, SSN College of Engineering Kalavakkam 603110, India
}

Correspondence should be addressed to Parvathy Ayalur Krishnamoorthy, akparvathy@hindustanuniv.ac.in

Received 10 May 2011; Revised 17 August 2011; Accepted 26 August 2011

Academic Editor: Derong Liu

Copyright () 2011 Parvathy Ayalur Krishnamoorthy et al. This is an open access article distributed under the Creative Commons Attribution License, which permits unrestricted use, distribution, and reproduction in any medium, provided the original work is properly cited.

In the exact linearization of involutive nonlinear system models, the issue of singularity needs to be addressed in practical applications. The approximate linearization technique due to Krener, based on Taylor series expansion, apart from being applicable to noninvolutive systems, allows the singularity issue to be circumvented. But approximate linearization, while removing terms up to certain order, also introduces terms of higher order than those removed into the system. To overcome this problem, in the case of quadratic linearization, a new concept called "generalized quadratic linearization" is introduced in this paper, which seeks to remove quadratic terms without introducing third- and higher-order terms into the system. Also, solution of generalized quadratic linearization of a class of control affine systems is derived. Two machine models are shown to belong to this class and are reduced to only linear terms through coordinate and state feedback. The result is applicable to other machine models as well.

\section{Introduction}

Control of nonlinear systems is gaining increasing attention in recent years due to its technical importance and its impact on various applications as well. When nonlinearities of systems become significant, linear control techniques generally fail to produce the desired results. However, feedback linearization of nonlinear systems allows linear control methods to be applied effectively to the resultant linearized system.

In the application of exact linearization technique [13 ] to involutive systems, an issue of singularity needs to be addressed in practical situations. The singularity is caused when a function of the system state tends towards zero causing the state feedback to fail. Approximate linearization technique due to Krener [4] based on a Taylor series expansion, applicable to non-involutive systems, has the merit of allowing the problem of singularity to be overcome.

Poincare [5] derived what are known as homological equations for approximate linearization of autonomous differential systems. Kang and Krener [6-8] extended the result for the approximate linearization of control affine systems and derived what are termed generalized homo- logical equations. Tall [9, 10] extended Kang and Krener's normal forms to multi-input systems. Devanathan $[11,12]$ developed necessary and sufficient conditions for quadratic linearization of control affine systems with a single input.

Using the approximate linearization technique, the problem of singularity of state feedback can be circumvented by appropriate definition of state feedback. But the approximate linearization, while removing nonlinearities up to a certain higher order, introduces nonlinearities into the system of higher order than those removed. Introduction of higher-order nonlinearities into a system which originally may not have such nonlinearities is a matter of concern.

In the case of machine models, for example, the secondorder dynamic models as given in [13] seem adequate to describe the normal behaviour of the machine. Applying quadratic linearization based on approximate linearization technique to this model introduces third- and higherorder terms into the model even though the machine does not possess nonlinearities of this order during normal operation.

In this paper, a new concept called "generalized quadratic linearization" which seeks to remove the second-order nonlinearity in the model without introducing third-and 
higher-order nonlinearities in the process is introduced. This is in contrast to the existing quadratic linearization techniques due to Kang and Krener [6-8] and Devanathan $[11,12]$ which introduce third- and-higher order terms in the process of removing second-order terms. The generalized quadratic linearization, being a stronger condition than quadratic linearization, imposes additional constraints on the quadratic polynomials of the system than those imposed due to quadratic linearization alone. Sufficient conditions on the quadratic polynomials together with coordinate and state feedback transformations are derived for a class of systems for which generalized quadratic linearization is applicable. In particular, the results are shown to apply to the induction motor and permanent magnet synchronous motor models. The results can be extended to other machine models as well. Application of generalized quadratic linearization to machine models also helps to avoid the issue of singularity which is a drawback attributed to the existing exact linearization of machine models $[2,3]$. This is the main contribution of the paper.

To summarise the rest of the paper, in Section 2, homological equations for arbitrary order linearization are introduced. Section 3 helps to simplify the homological equations for quadratic linearization, thus paving the way for the central result on generalized quadratic linearization presented in Section 4. In Section 5, generalized quadratic linearization is applied to two machine models including a real-world numerical example. In Section 6, the paper is concluded.

\section{Background}

Consider a multiple-input control affine system of the form $[7,14,15]$

$$
\begin{aligned}
\dot{x}= & A x+B u+f^{(2)}(x)+f^{(3)}(x)+\cdots+f^{(p)}(x) \\
& +\cdots+g^{(1)}(x) u+g^{(2)}(x) u+\cdots+g^{(p-1)}(x) u+\cdots,
\end{aligned}
$$

where $(A, B)$ are in Brunovsky normal form. That is, let $k=$ $\left\{k_{1}, k_{2}, \ldots, k_{r}\right\}$ be a sequence of integers such that $k_{1} \geq k_{2} \geq$ $\cdots \geq k_{r}$ such that $k_{1}+k_{2}+\cdots+k_{r}=n$

$$
\begin{gathered}
A=\left[\begin{array}{cccc}
A_{k_{1}} & 0 & \cdots & 0 \\
0 & A_{k_{2}} & \cdots & 0 \\
\vdots & \vdots & \vdots & \vdots \\
0 & 0 & \cdots & A_{k_{r}}
\end{array}\right], \\
B=\left[\begin{array}{ccccccc}
b_{k_{1}} & 0 & \cdots & 0 & 0 & \cdots & 0 \\
0 & b_{k_{2}} & \cdots & 0 & 0 & \cdots & 0 \\
\vdots & \vdots & \vdots & \vdots & \vdots & \vdots \\
0 & 0 & \cdots & b_{k_{r}} & 0 & \cdots & 0
\end{array}\right],
\end{gathered}
$$

where matrix $A$ is partitioned into $r^{2}$ blocks while matrix $B$ is partitioned into $\mu r$ blocks with $r \leq \mu$. Each block $A_{k_{i}}$ or $b_{k_{i}}$ is of the form

$$
A_{k_{i}}=\left[\begin{array}{ccccc}
0 & 1 & 0 & \cdots & 0 \\
0 & 0 & 1 & \cdots & 0 \\
\vdots & \vdots & \vdots & \vdots & \vdots \\
0 & 0 & 0 & \cdots & 1 \\
0 & 0 & 0 & \cdots & 0
\end{array}\right], \quad b_{k_{i}}=\left[\begin{array}{c}
0 \\
0 \\
\vdots \\
0 \\
1
\end{array}\right] .
$$

$A_{k_{i}}$ is of order $k_{i} \times k_{i}, b_{k_{i}}$ is of order $k_{i} \times 1$, and $B$ is $n \times \mu$ matrix. $x=\left[x_{1}, x_{2}, \ldots, x_{n}\right]^{T}$ and $u=\left[u_{1}, u_{2}, \ldots, u_{\mu}\right]^{T}$ are the vector input such that $\mu<n$. $f^{(p)}(x)$ and $g^{(p-1)}(x)$ are vector polynomials of order $p$ and $(p-1)$, respectively, $p=2,3, \ldots$.

In order to linearize the system, change of coordinate and state feedback [6] of the following form is considered:

$$
\begin{gathered}
y=x+\phi(x), \\
u=\left(I_{\mu}+\beta(x)\right) v+\alpha(x),
\end{gathered}
$$

where

$$
\begin{gathered}
\phi(x)=\sum_{j=2,3,4, \ldots} \phi^{(j)}(x), \quad \alpha(x)=\sum_{j=2,3,4, \ldots} \alpha^{(j)}(x), \\
\beta(x)=\sum_{j=2,3,4, \ldots} \beta^{(j-1)}(x),
\end{gathered}
$$

and $I_{\mu}$ is the identity matrix of order $\mu$. Applying the transformations (4), (1) is reduced to

$$
\dot{y}=A y+B v
$$

provided the following equations, called homological equations [6], are satisfied for $m \geq 2$ :

$$
\begin{aligned}
& -A \phi^{(m)}(x)+B \alpha^{(m)}(x)+f^{\prime(m)}(x)+\frac{\partial \phi^{(m)}(x)}{\partial x} A x=0, \\
& B \beta^{(m-1)}(x) v+\frac{\partial \phi^{(m)}(x)}{\partial x} B v+g^{\prime(m-1)}(x) v=0, \quad \forall v,
\end{aligned}
$$

where $f^{\prime(m)}(x)=f^{(m)}(x)$ for $m=2$ and $f^{\prime(m)}(x)$ is expressed in terms of $f^{(m-i)}(x), i=0,1,2, \ldots(m-2)$, and $\phi^{(m-j)}(x)$, $j=1,2, \ldots(m-2), m>2 . g^{(m-1)}(x)=g^{(1)}(x) ; m=2$, and $g^{\prime(m-1)}(x)$ is expressed in terms of $g^{(m-i)}(x), i=1,2, \ldots(m-$ $1)$, and $\phi^{(m-j)}(x), j=1,2, \ldots(m-2), m>2$.

\section{Quadratic Linearization}

Consider the specialized case of system (1) containing only quadratic terms in $x$ as in

$$
\dot{x}=A x+B u+f^{(2)}(x) .
$$

Quadratic linearization of (9) involves specialization of the result discussed in Section 2 for $m=2$ and simplifying it 
by applying $g^{(1)}(x)=0$. That is, applying the following coordinate transformation and feedback,

$$
\begin{gathered}
y=x+\phi^{(2)}(x), \\
u=\left(I_{\mu}+\beta^{(1)}(x)\right) v+\alpha^{(2)}(x),
\end{gathered}
$$

(9) is reduced to

$$
\dot{y}=A y+B v+O^{(3)}(y, v),
$$

where $O^{(3)}(y, v)$ corresponds to terms of order 3 or higher, provided the following equations are satisfied:

$$
\begin{gathered}
-A \phi^{(2)}(x)+B \alpha^{(2)}(x)+f^{(2)}(x)+\frac{\partial \phi^{(2)}(x)}{\partial x} A x=0, \\
B \beta^{(1)}(x) v+\frac{\partial \phi^{(2)}(x)}{\partial x} B v=0, \quad \forall v .
\end{gathered}
$$

Remark 3.1. Applying quadratic linearization to system (9) introduces third- and higher-order terms into the system (see (11)) even though originally the system does not possess nonlinearities of this order. To avoid this situation, we introduce a new concept of generalized quadratic linearization which seeks to remove the second-order nonlinearity in the system without introducing third- and higher-order nonlinearities in the process. Sufficient conditions for the generalized quadratic linearization for a class of systems are discussed in the next section.

We next simplify the homological equations (12) and (13) by representing them in partitioned form, using the Brunovsky normal form introduced in Section 2.

Let

$$
\begin{gathered}
\phi^{(2)}(x)=\left[\begin{array}{c}
\phi_{k_{1}}^{(2)}(x) \\
\phi_{k_{2}}^{(2)}(x) \\
\vdots \\
\phi_{k_{r}}^{(2)}(x)
\end{array}\right], \\
x=\left[\begin{array}{c}
x_{k_{1}} \\
x_{k_{2}} \\
\vdots \\
x_{k_{r}}
\end{array}\right], \\
f^{(2)}(x)=\left[\begin{array}{c}
f_{k_{1}}^{(2)}(x) \\
f_{k_{2}}^{(2)}(x) \\
\vdots \\
f_{k_{r}}^{(2)}(x)
\end{array}\right], \\
\alpha^{(2)}(x)=\left[\begin{array}{c}
\alpha_{1}^{(2)}(x) \\
\alpha_{2}^{(2)}(x) \\
\vdots \\
\alpha_{\mu}^{(2)}(x)
\end{array}\right],
\end{gathered}
$$

where putting $p_{i}=\sum^{i} k_{i}, i=1,2, \ldots r$,

$$
\begin{aligned}
& \phi_{k_{i}}^{(2)}(x)=\left[\begin{array}{c}
\phi_{p_{i-1}+1}^{(2)}(x) \\
\vdots \\
\phi_{p_{i-1}+t}^{(2)}(x) \\
\vdots \\
\phi_{p_{i}-1}^{(2)}(x) \\
\phi_{p_{i}}^{(2)}(x)
\end{array}\right], \\
& x_{k_{i}}=\left[\begin{array}{c}
x_{p_{i-1}+1} \\
\vdots \\
x_{p_{i-1}+t} \\
\vdots \\
x_{p_{i}-1} \\
x_{p_{i}}
\end{array}\right] \text {, } \\
& f_{k_{i}}^{(2)}(x)=\left[\begin{array}{c}
f_{p_{i-1}+1}^{(2)}(x) \\
\vdots \\
f_{p_{i-1}+t}^{(2)}(x) \\
\vdots \\
f_{p_{i}-1}(x) \\
f_{p_{i}}^{(2)}(x)
\end{array}\right] \text {, } \\
& \frac{\partial \phi^{(2)}(x)}{\partial x}=\left[\begin{array}{cccc}
\phi_{k_{1}, k_{1}}^{(2)} & \phi_{k_{1}, k_{2}}^{(2)} & \cdots & \phi_{k_{1}, k_{r}}^{(2)} \\
\phi_{k_{2}, k_{1}}^{(2)} & \phi_{k_{2}, k_{2}}^{(2)} & \cdots & \phi_{k_{2}, k_{r}}^{(2)} \\
\vdots & \vdots & \vdots & \vdots \\
\phi_{k_{r}, k_{1}}^{(2)} & \phi_{k_{r}, k_{2}}^{(2)} & \cdots & \phi_{k_{r}, k_{r}}^{(2)}
\end{array}\right],
\end{aligned}
$$

where

$$
\phi_{k_{i}, k_{j}}^{(2)}=\left[\begin{array}{ccc}
\frac{\partial \phi_{p_{i-1}+1}^{(2)}(x)}{\partial x_{p_{j-1}+1}} & \cdots & \frac{\partial \phi_{p_{i-1}+1}^{(2)}(x)}{\partial x_{p_{j}}} \\
\vdots & \vdots & \vdots \\
\frac{\partial \phi_{p_{i-1}+t}^{(2)}(x)}{\partial x_{p_{j-1}+1}} & \cdots & \frac{\partial \phi_{p_{i-1}+t}^{(2)}(x)}{\partial x_{p_{j}}} \\
\vdots & \vdots & \vdots \\
\frac{\partial \phi_{p_{i}}^{(2)}(x)}{\partial x_{p_{j-1}+1}} & \cdots & \frac{\partial \phi_{p_{i}}^{(2)}(x)}{\partial x_{p_{j}}}
\end{array}\right], \quad i, j=1,2, \ldots r,
$$


where

$$
\beta_{i}^{(1)}(x)=\left[\beta_{i, 1}^{(1)}(x), \beta_{i, 2}^{(1)}(x), \ldots, \beta_{i, \mu}^{(1)}(x)\right], \quad i=1,2, \ldots, \mu .
$$

Using (2), (14)-(17), and (21), the ith block row of (12) can be written as

$$
\begin{array}{r}
-A_{k_{i}} \phi_{k_{i}}^{(2)}(x)+b_{k_{i}} \alpha_{i}^{(2)}(x)+f_{k_{i}}^{(2)}(x)+\sum_{j=1}^{r} \phi_{k_{i}, k_{j}} A_{k_{j}} x_{k_{j}}=0 \\
i=1,2, \ldots, r .
\end{array}
$$

The second homological equation, namely, (13), can be simplified as follows. Since the effect of control input $u$ in (9) is dependent on the $B$ matrix only, without loss of generality, we can then assume that $\mu=r$, because the terms in $B$ matrix are nonzero only for the first $r$ inputs. So, we can write the revised $B$ matrix as

$$
B=\left[\begin{array}{cccc}
b_{k_{1}} & 0 & \cdots & 0 \\
0 & b_{k_{2}} & \cdots & 0 \\
\vdots & \vdots & \vdots & \vdots \\
0 & 0 & \cdots & b_{k_{r}}
\end{array}\right] .
$$

We now put (13) in partitioned form. Using (2) and (21)-(23), the $i$ th block row of (13) can be written as

$$
\begin{array}{r}
b_{k_{i}} \beta_{i}^{(1)}(x) v+\left[\begin{array}{lll}
\phi_{k_{i}, k_{1}} b_{k_{1}} & \phi_{k_{i}, k_{2}} b_{k_{2}} \cdots & \phi_{k_{i}, k_{r}} b_{k_{r}}
\end{array}\right] v=0 \\
\forall v, i=1,2, \ldots, r .
\end{array}
$$

Since (26) is to be an identity in $v$, we can drop $v$ and rewrite (26) as

$$
\begin{array}{ccccc}
0 & 0 & \cdots & 0 \\
0 & 0 & \cdots & 0 \\
0 & 0 & \cdots & 0 \\
\vdots & \vdots & \vdots & \vdots \\
\vdots & \vdots & \vdots & \vdots \\
0 & 0 & \cdots & 0 \\
\beta_{i, 1}^{(1)}(x) & \left.\begin{array}{cccc}
\beta_{i, 2}^{(1)}(x) & \cdots & \beta_{i, r}^{(1)}(x)
\end{array}\right] \\
+ & {\left[\begin{array}{ccc}
\frac{\partial \phi_{p_{i-1}+1}^{(2)}(x)}{\partial x_{p_{1}}} & \cdots & \frac{\partial \phi_{p_{i-1}+1}^{(2)}(x)}{\partial x_{p_{r}}} \\
\vdots & & \vdots \\
\frac{\partial \phi_{p_{i-1}+t}^{(2)}(x)}{\partial x_{p_{1}}} & \cdots & \frac{\partial \phi_{p_{i-1}+t}^{(2)}(x)}{\partial x_{p_{r}}} \\
\vdots & & \vdots \\
\frac{\partial \phi_{p_{i}}^{(2)}(x)}{\partial x_{p_{1}}} & \cdots & \frac{\partial \phi_{p_{i}}^{(2)}(x)}{\partial x_{p_{r}}}
\end{array}\right]=0} \\
& \cdots & i=1,2, \ldots, r .
\end{array}
$$

Since $\beta_{i, l}^{(1)}(x), \quad l=1,2, \ldots, r$, are arbitrary, it is clear that a necessary condition for (13) to be satisfied is

$$
\begin{array}{r}
\frac{\partial \phi_{p_{i-1}+t}^{(2)}(x)}{\partial x_{p_{q}}}=0, \quad q=1,2, \ldots, r, t=1,2, \ldots,\left(k_{i}-1\right), \\
i=1,2, \ldots, r .
\end{array}
$$

\section{Generalized Quadratic Linearization}

Theorem 4.1. Consider the system

$$
\dot{x}=A x+B u+f^{(2)}(x)
$$

where $x=\left[x_{1}, x_{2}, \ldots, x_{n}\right]^{T}$ and $u=\left[u_{1}, u_{2}, \ldots, u_{r}\right]^{T}, n>r$. $(A, B)$ are in Brunovsky normal form as given in (2), (3), and (25). $f^{(2)}(x)$ is given by (16), where

$$
f_{k_{i}}^{(2)}(x)=\left\{\begin{array}{c}
{\left[\begin{array}{c}
0 \\
\vdots \\
0 \\
f_{p_{i}-1}^{(2)}\left(x^{\prime}\right) \\
f_{p_{i}}^{(2)}(x)
\end{array}\right], \quad k_{i}>2,} \\
{\left[\begin{array}{c}
f_{p_{i}-1}^{(2)}\left(x^{\prime}\right) \\
f_{p_{i}}^{(2)}(x)
\end{array}\right], \quad k_{i}=2,} \\
{\left[\begin{array}{c}
\left.f_{p_{i}}^{(2)}(x)\right], \\
i=1,2, \ldots r,
\end{array}\right.}
\end{array}\right.
$$

$f_{k_{i}}^{(2)}(x)$ is of order $k_{i} \times 1$ and $x^{\prime}$ is given by the r-tuple $x^{\prime}=$ $\left(x_{p_{1}}, x_{p_{2}}, \ldots, x_{p_{r}}\right)$. Then the transformation $(4)$, where $\phi(x)=$ $\phi^{(2)}(x)$, is given by (14), where

$$
\begin{aligned}
& \phi_{k_{i}}^{(2)}(x)=\left\{\begin{array}{c}
{\left[\begin{array}{c}
\phi_{p_{i-1}+1}^{(2)}(x) \\
\vdots \\
\phi_{p_{i}-1}^{(2)}(x) \\
\phi_{p_{i}}^{(2)}(x)
\end{array}\right]=\left[\begin{array}{c}
0 \\
\vdots \\
0 \\
f_{p_{i}-1}^{(2)}\left(x^{\prime}\right)
\end{array}\right], \quad k_{i} \geq 2,} \\
{\left[\phi_{p_{i}}^{(2)}\left(x^{\prime}\right)\right] ; \quad \phi_{p_{i}}^{(2)}\left(x^{\prime}\right) \text { arbitrary, } \quad k_{i}=1,}
\end{array}\right. \\
& i=1,2, \ldots, r
\end{aligned}
$$

$$
\begin{aligned}
& \alpha(x)=\alpha^{(2)}(x) \text { given by }(17) \text {, where } \\
& \qquad \alpha_{i}^{(2)}(x)=-f_{p_{i}}^{(2)}(x), \quad i=1,2, \ldots, r, \\
& \beta^{(m-1)}(x)=(-1)^{m-1}\left\{B^{T} \frac{\partial \phi^{(2)}(x)}{\partial x} B\right\}^{m-1}, \quad m \geq 2,
\end{aligned}
$$


reduces the system (29) to

$$
\dot{y}=A y+B v .
$$

Proof. We first show that the quadratic linearization of (29) is achieved using (10), assuming (30)-(33). That is, it needs to be verified that (12) and (13) are satisfied. To verify that (12) is satisfied, it is sufficient to verify that (24) is satisfied. Substituting (30)-(32) into (24), we need to satisfy the following:

$$
-\left[\begin{array}{ccccc}
0 & 1 & 0 & \cdots & 0 \\
0 & 0 & 1 & \cdots & 0 \\
\vdots & \vdots & \vdots & \vdots & \vdots \\
0 & 0 & 0 & \cdots & 1 \\
0 & 0 & 0 & \cdots & 0
\end{array}\right]\left[\begin{array}{c}
0 \\
0 \\
\vdots \\
0 \\
f_{p_{i}-1}^{(2)}\left(x^{\prime}\right)
\end{array}\right]
$$

$$
-\left[\begin{array}{c}
0 \\
0 \\
\vdots \\
0 \\
1
\end{array}\right] f_{p_{i}}^{(2)}(x)+\left[\begin{array}{c}
0 \\
\vdots \\
0 \\
f_{p_{i}-1}^{(2)}\left(x^{\prime}\right) \\
f_{p_{i}}^{(2)}(x)
\end{array}\right]
$$$$
+\sum_{j=1}^{r}\left[\begin{array}{ccc}
0 & \cdots & 0 \\
\vdots & \vdots & \vdots \\
0 & \cdots & 0 \\
\frac{\partial f_{p_{i}-1}^{(2)}\left(x^{\prime}\right)}{\partial x_{p_{j-1}+1}} & \cdots & \frac{\partial f_{p_{i}-1}^{(2)}\left(x^{\prime}\right)}{\partial x_{p_{j}}}
\end{array}\right]\left[\begin{array}{c}
x_{p_{j-1}+2} \\
x_{p_{j-1}+3} \\
\vdots \\
x_{p_{j}} \\
0
\end{array}\right]=0 \text {, }
$$

$$
i=1,2, \ldots, r \text {. }
$$

Note that $f_{p_{i}-1}^{(2)}\left(x^{\prime}\right)$ is a function of $x_{p_{j}}, \quad j=1,2, \ldots, r$, only. Hence, $\partial f_{p_{i}-1}^{(2)}\left(x^{\prime}\right) /\left(\partial x_{p_{j-1}+t}\right)=0, \quad t=1,2, \ldots,\left(k_{i}-1\right)$. Equation (35) then reduces to the following after simplification:

$$
\begin{aligned}
& {\left[\begin{array}{c}
0 \\
0 \\
\vdots \\
f_{p_{i}-1}^{(2)}\left(x^{\prime}\right) \\
0
\end{array}\right]-\left[\begin{array}{c}
0 \\
0 \\
\vdots \\
0 \\
f_{p_{i}}^{(2)}(x)
\end{array}\right]+\left[\begin{array}{c}
0 \\
\vdots \\
0 \\
f_{p_{i}-1}^{(2)}\left(x^{\prime}\right) \\
f_{p_{i}}^{(2)}(x)
\end{array}\right]} \\
& +\sum_{j=1}^{r}\left[\begin{array}{cccc}
0 & \cdots & 0 \\
\vdots & \vdots & \vdots & \vdots \\
\vdots & \vdots & \vdots & \vdots \\
0 & \cdots & 0 & \frac{\partial f_{p_{i}-1}^{(2)}\left(x^{\prime}\right)}{\partial x_{p_{j}}}
\end{array}\right]\left[\begin{array}{c}
x_{p_{j-1}+2} \\
x_{p_{j-1}+3} \\
\vdots \\
x_{p_{j}} \\
0
\end{array}\right]=0, \quad i=1,2, \ldots, r .
\end{aligned}
$$

Clearly (36) is an identity. Hence (24) is satisfied for $k_{i} \geq 2$. For the case $k_{i}=1$, it is easy to verify that (24) is satisfied as $\phi_{p_{i}}^{(2)}\left(x^{\prime}\right)$ is a function of $x_{p_{j}}, \quad j=1,2, \ldots, r$, only.
Equation (28) is a necessary condition for (13) to hold, and it follows easily from $(31)$, where $\phi_{p_{i-1}+t}^{(2)}(x)=0, t=$ $1,2, \ldots,\left(k_{i}-1\right), i=1,2, \ldots, r$. To satisfy (13), note that, from (25), it follows that

$$
B^{T} B=I_{r} .
$$

Premultiply (13) by $B^{T}$ and rearrange to yield

$$
\beta^{(1)}(x)=-B^{T} \frac{\partial \phi^{(2)}(x)}{\partial x} B
$$

which is (33) for $m=2$. Substitution of (38) satisfies (13). Hence, the result holds for $m=2$.

As a result of quadratic linearization (i.e., satisfying (12) and (13)), higher-order terms are left in the system as per (11) even though originally (29) did not have third- and higher-order terms. These are now to be removed to effect generalized quadratic linearization. The higher-order terms are functions of $\alpha(x), \phi(x)$, and $\beta(x)$ and are given for the case $g^{(m)}(x)=0, m \geq 1$, through algebraic computations as

$$
\begin{array}{r}
f^{\prime(m)}(x)=\frac{\partial \phi^{(m-1)}(x)}{\partial x} f^{(2)}(x)+\sum_{j=2}^{m-1} \frac{\partial \phi^{(j)}(x)}{\partial x} B \alpha^{(m-j+1)}(x), \\
m \geq 3, \\
g^{\prime(m-1)}(x)=\sum_{j=2}^{m-1} \frac{\partial \phi^{(j)}(x)}{\partial x} B \beta^{(m-j)}(x), \quad m>2 .
\end{array}
$$

For $m=3$,

$$
f^{\prime(3)}(x)=\frac{\partial \phi^{(2)}(x)}{\partial x}\left[f^{(2)}(x)+B \alpha^{(2)}(x)\right] .
$$

Partitioning $f^{\prime(3)}(x)$ similar to $f^{(2)}(x)$ in (16) and considering the $i$ th block row,

$$
\begin{aligned}
& f_{k_{i}}^{\prime(3)}(x)=\sum_{j=1}^{r} \phi_{k_{i}, k_{j}}\left(f_{k_{j}}^{(2)}(x)+b_{k_{j}} \alpha_{j}^{(2)}(x)\right) \\
& =\sum_{j=1}^{r}\left(\left[\begin{array}{ccc}
\frac{\partial \phi_{p_{i-1}+1}^{(2)}(x)}{\partial x_{p_{j-1}+1}} & \cdots & \frac{\partial \phi_{p_{i-1}+1}^{(2)}(x)}{\partial x_{p_{j}}} \\
\vdots & \vdots & \vdots \\
\frac{\partial \phi_{p_{i-1}+t}^{(2)}(x)}{\partial x_{p_{j-1}+1}} & \cdots & \frac{\partial \phi_{p_{i-1}+t}^{(2)}(x)}{\partial x_{p_{j}}} \\
\vdots & \vdots & \vdots \\
\frac{\partial \phi_{p_{i}}^{(2)}(x)}{\partial x_{p_{j-1}+1}} & \cdots & \frac{\partial \phi_{p_{i}}^{(2)}(x)}{\partial x_{p_{j}}}
\end{array}\right]\right. \\
& \left.\times\left[\begin{array}{c}
0 \\
0 \\
\vdots \\
\vdots \\
0 \\
f_{p_{j}-1}^{(2)}\left(x^{\prime}\right) \\
f_{p_{j}}^{(2)}(x)+\alpha_{j}^{(2)}(x)
\end{array}\right]\right), \quad i=1,2, \ldots, r .
\end{aligned}
$$


Using (31) and (32) and that $\phi_{p_{i}}^{(2)}(x)$ being a function of $x_{p_{j}}, j=1,2, \ldots, r$, only as above, $(42)$ can be reduced to

$$
f_{k_{i}}^{\prime(3)}(x)=\sum_{j=1}^{r}\left[\begin{array}{cccc}
0 & \cdots & 0 & 0 \\
\vdots & \vdots & \vdots & \vdots \\
\vdots & \vdots & \vdots & \vdots \\
0 & \cdots & 0 & \frac{\partial f_{p_{i}-1}^{(2)}\left(x^{\prime}\right)}{\partial x_{p_{j}}}
\end{array}\right]\left[\begin{array}{c}
0 \\
\vdots \\
0 \\
f_{p_{j}-1}^{(2)}\left(x^{\prime}\right) \\
0
\end{array}\right]=0,
$$

Hence, $f^{\prime(3)}(x)=0$. Homological equation (7), for $m=3$, is satisfied if we put $\phi^{(3)}(x)=0$ and $\alpha^{(3)}(x)=0$.

For $m=4$,

$$
\begin{aligned}
f^{\prime(4)}(x)= & \frac{\partial \phi^{(3)}(x)}{\partial x} f^{(2)}(x)+\frac{\partial \phi^{(2)}(x)}{\partial x} B \alpha^{(3)}(x) \\
& +\frac{\partial \phi^{(3)}(x)}{\partial x} B \alpha^{(2)}(x) .
\end{aligned}
$$

As $\phi^{(3)}(x)=0$ and $\alpha^{(3)}(x)=0$, it follows from (44) that $f^{\prime(4)}(x)=0$. Hence, one can have $\phi^{(4)}(x)=0$ and $\alpha^{(4)}(x)=$ 0 to satisfy (7) for $m=4$. Proceeding this way, it can be proved that $f^{\prime(m)}(x)=0, m>3$.

Noting that from $(40), g^{\prime(2)}(x)=\left(\partial \phi^{(2)}(x) / \partial x\right) B \beta^{(1)}(x)$ for $m=3$, and (8) can be written as

$$
B \beta^{(2)}(x)+\frac{\partial \phi^{(3)}(x)}{\partial x} B+g^{\prime(2)}(x)=0,
$$

from which using the fact that $\phi^{(3)}(x)=0, \beta^{(2)}(x)=$ $-B^{T}\left(\partial \phi^{(2)}(x) / \partial x\right) B \beta^{(1)}(x)=\left(B^{T}\left(\partial \phi^{(2)}(x) / \partial x\right) B\right)^{2}$ using (38) is derived. Substitution of $\beta^{(2)}(x)$ satisfies (8) for $m=3$.

Proceeding this way, one can show that $f^{\prime(m)}=0, m>3$, and we can choose $\phi^{(m)}=0$ and $\alpha^{(m)}=0, m>3$, to satisfy (7). $\beta^{(m-1)}(x)$ as given in (33) is easily derived so as to satisfy (8), where $g^{\prime(m-1)}(x)$ is given in (40), using the same technique as for $m=2,3$ successively as above, hence the result.

Remark 4.2. The result of Theorem 4.1 provides a solution to the generalized quadratic linearization of a class of systems defined by (29). Equation (29) may appear to be a strong condition. But this is needed because of the requirement that the third- and all higher-order nonlinearities, which are usually introduced into the system during the course of quadratic linearization, identically vanish. As shown in Section 5, system (29) can represent many electric machine models [13] of which induction motor and permanent magnet synchronous motor are examples. Also, temperature and flow variables in heat transfer (composition and flow variables in mass transfer) processes, for example, combine to produce quadratic nonlinearity [16] of the form (9) which is a general form of (29). Further, even if a quadratic model (9) does not correspond exactly to model (29), one can always tune the coordinate and state feedback on the lines described by the authors in [17] to bring the system to a linear normal form. It can also be pointed out that quadratic system of the form $\dot{x}=A x+B u+f^{(2)}(x)+g^{(1)}(x) u$ can be brought to the form $\dot{x}=A x+B v+f^{\prime(2)}(x)$ through state feedback as shown in [6]. Hence the forms (9) and (29), which are a special case of (9), are more general than they appear to be.

Remark 4.3. A specialized result of Theorem 4.1 appears in an earlier paper by the authors [18]. In [18], the concept of generalized quadratic linearization is applied to a class of control affine systems with a specific configuration of Brunovsky form for multiple inputs. A PMSM model is shown to belong to this class and the generalized quadratic linearization of PMSM is derived. In Theorem 4.1, however, we have considered a more general class of systems with no assumption on the configuration of the Brunovsky form for multiple inputs. Hence, the theoretical result of [18] is a special case of the result of Theorem 4.1.

\section{Generalized Quadratic Linearization of Machine Models}

5.1. Squirrel Cage Induction Motor. The squirrel cage induction motor model can be derived as below [13]:

$$
\dot{x}=A x+B u+f^{(2)}(x)
$$

where $x=\left[x_{1}, x_{2}, x_{3}, x_{4}, x_{5}\right]^{T}=\left[i_{\mathrm{ds}}^{s}, i_{\mathrm{qs}}^{s}, \psi_{\mathrm{dr}}^{s}, \psi_{\mathrm{qr}}^{s}, \omega_{r}\right]^{T}, u=$ $\left[u_{1}, u_{2}, u_{3}, u_{4}\right]^{T}=\left[v_{\mathrm{ds}}^{s}, v_{\mathrm{qs}}^{s}, \psi^{\prime}, \hat{\psi}_{\mathrm{dr}}^{s}\right]$, where $\psi^{\prime}=i_{\mathrm{qs}}^{s^{*}} \widehat{\psi}_{\mathrm{dr}}^{s}-$ $i_{\mathrm{ds}}^{s^{*}} \hat{\psi}_{\mathrm{qr}}^{s}$, where $v_{\mathrm{qs}}^{s}, v_{\mathrm{ds}}^{s}, i_{\mathrm{qs}}^{s}$, and $i_{\mathrm{ds}}^{s}$ represent the quadrature and direct axis voltages and currents, respectively, and $\psi_{\mathrm{dr}}^{s}, \psi_{\mathrm{qr}}^{s}$ represent direct and quadrature axis fluxes, respectively. $\hat{\psi}_{\mathrm{dr}}^{s}, \hat{\psi}_{\mathrm{qr}}^{s}$ represent the estimated direct and quadrature flux, respectively, and $\omega_{r}$ represents the angular velocity of the rotor. $i_{\mathrm{qs}}^{s^{*}}$ and $i_{\mathrm{ds}}^{*}$ represent the reference values of quadrature and direct axis currents, respectively. The superscript refers to variables in the stationary frame:

$A=\left[\begin{array}{ccccc}-\frac{\left(L_{m}^{2} R_{r}+L_{r}^{2} R_{s}\right)}{\sigma L_{s} L_{r}^{2}} & 0 & \frac{L_{m} R_{r}}{\sigma L_{s} L_{r}^{2}} & 0 & 0 \\ 0 & -\frac{\left(L_{m}^{2} R_{r}+L_{r}^{2} R_{s}\right)}{\sigma L_{s} L_{r}^{2}} & 0 & \frac{L_{m} R_{r}}{\sigma L_{s} L_{r}^{2}} & 0 \\ \frac{L_{m} R_{r}}{L_{r}} & 0 & 0 & 0 & 0 \\ 0 & \frac{L_{m} R_{r}}{L_{r}} & 0 & -\frac{R_{r}}{L_{r}} & 0 \\ 0 & 0 & 0 & 0 & 0\end{array}\right]$, 


$$
f^{(2)}(x)=\left[\begin{array}{c}
\frac{L_{m} \omega_{r} \psi_{\mathrm{qr}}^{s}}{\sigma L_{s} L_{r}} \\
-\frac{L_{m} \omega_{r} \psi_{\mathrm{dr}}^{s}}{\sigma L_{s} L_{r}} \\
-\omega_{r} \psi_{\mathrm{qr}}^{s} \\
\omega_{r} \psi_{\mathrm{dr}}^{s} \\
0
\end{array}\right]=\left[\begin{array}{c}
c_{1} x_{4} x_{5} \\
-c_{1} x_{3} x_{5} \\
-x_{4} x_{5} \\
x_{3} x_{5} \\
0
\end{array}\right],
$$

where $L_{m}, L_{r}$, and $L_{s}$ represent the magnetizing, rotor, and stator inductances, respectively. $R_{r}$ and $R_{s}$ represent the rotor and stator resistances, respectively. $J$ represents the system moment of inertia, and $P$ represents the number of poles. $\sigma=1-L_{m}^{2} / L_{s} L_{r}$ and $c_{1}=L_{m} / \sigma L_{s} L_{r}$. The model (46) can be reduced to normal form in a standard way using linear transformation of state variables [19] and linear state feedback as follows, where $x, u, A, B$ and $f^{(2)}(x)$ are retained for simplicity of notation:

$$
\dot{x}=A x+B u+f^{(2)}(x),
$$

where $A$ and $B$ given by (2), where $r=\mu=4$ and $k_{1}=$ 2, $k_{2}=k_{3}=k_{4}=1$, are

$$
\begin{gathered}
A=\left[\begin{array}{lllll}
0 & 1 & 0 & 0 & 0 \\
0 & 0 & 0 & 0 & 0 \\
0 & 0 & 0 & 0 & 0 \\
0 & 0 & 0 & 0 & 0 \\
0 & 0 & 0 & 0 & 0
\end{array}\right], \quad B=\left[\begin{array}{llll}
0 & 0 & 0 & 0 \\
1 & 0 & 0 & 0 \\
0 & 1 & 0 & 0 \\
0 & 0 & 1 & 0 \\
0 & 0 & 0 & 1
\end{array}\right], \\
f^{(2)}(x)=\left[\begin{array}{l}
f_{1}^{(2)}(x) \\
f_{2}^{(2)}(x) \\
f_{3}^{(2)}(x) \\
f_{4}^{(2)}(x) \\
f_{5}^{(2)}(x)
\end{array}\right],
\end{gathered}
$$

where $f_{1}^{(2)}(x)=f_{1,35} x_{3} x_{5}+f_{1,45} x_{4} x_{5}, f_{2}^{(2)}(x)=f_{2,35} x_{3} x_{5}+$ $f_{2,45} x_{4} x_{5}, f_{3}^{(2)}(x)=f_{3,35} x_{3} x_{5}+f_{3,45} x_{4} x_{5}, f_{4}^{(2)}(x)=f_{4,35} x_{3} x_{5}+$ $f_{4,45} x_{4} x_{5}, f_{5}^{(2)}(x)=f_{5,35} x_{3} x_{5}+f_{5,45} x_{4} x_{5}$.

Remark 5.1. $f^{(2)}(x)$ can be partitioned using (16) as

$$
f^{(2)}(x)=\left[\begin{array}{l}
f_{k_{1}}^{(2)}(x) \\
f_{k_{2}}^{(2)}(x) \\
f_{k_{3}}^{(2)}(x) \\
f_{k_{4}}^{(2)}(x)
\end{array}\right],
$$

where

$$
\begin{aligned}
& f_{k_{1}}^{(2)}(x)=\left[\begin{array}{l}
f_{1}^{(2)}(x) \\
f_{2}^{(2)}(x)
\end{array}\right]=\left[\begin{array}{l}
f_{1,35} x_{3} x_{5}+f_{1,45} x_{4} x_{5} \\
f_{2,35} x_{3} x_{5}+f_{2,45} x_{4} x_{5}
\end{array}\right], \\
& f_{k_{2}}^{(2)}(x)=\left[f_{3}^{(2)}(x)\right]=\left[f_{3,35} x_{3} x_{5}+f_{3,45} x_{4} x_{5}\right] \text {, } \\
& f_{k_{3}}^{(2)}(x)=\left[f_{4}^{(2)}(x)\right]=\left[f_{4,35} x_{3} x_{5}+f_{4,45} x_{4} x_{5}\right], \\
& f_{k_{4}}^{(2)}(x)=\left[f_{5}^{(2)}(x)\right]=\left[f_{5,35} x_{3} x_{5}+f_{5,45} x_{4} x_{5}\right] .
\end{aligned}
$$

As $f_{1}^{(2)}(x)$ is a function of $x_{3}, x_{4}$, and $x_{5}$ only and hence a function of $x^{\prime}=\left(x_{2}, x_{3}, x_{4}, x_{5}\right)$, one can write $f_{1}^{(2)}(x)=$ $f_{1}^{(2)}\left(x^{\prime}\right)$. The systems given by (48) and (49) are then in line with conditions of Theorem 4.1 as given in (30). Hence, Theorem 4.1 can be applied to system (48) to obtain the solution of generalized quadratic linearization which is given in Corollary 5.2.

Corollary 5.2. The system (48) can be quadratic linearized in the generalized sense using the transformation

$$
\begin{gathered}
y=x+\phi(x), \\
u=\left(I_{4}+\beta(x)\right) v+\alpha(x),
\end{gathered}
$$

where

$$
\begin{gathered}
\phi(x)=\phi^{(2)}(x)=\left[\begin{array}{c}
0 \\
f_{1}^{(2)}\left(x^{\prime}\right) \\
\phi_{3}^{(2)}\left(x^{\prime}\right) \\
\phi_{4}^{(2)}\left(x^{\prime}\right) \\
\phi_{5}^{(2)}\left(x^{\prime}\right)
\end{array}\right], \\
\alpha(x)=\alpha^{(2)}(x)=\left[\begin{array}{c}
-f_{2}^{(2)}(x) \\
-f_{3}^{(2)}(x) \\
-f_{4}^{(2)}(x) \\
-f_{5}^{(2)}(x)
\end{array}\right], \\
\beta^{(m-1)}(x)=(-1)^{m-1}\left\{B^{T} \frac{\partial \phi^{(2)}(x)}{\partial x} B\right\}^{m-1},
\end{gathered}
$$

where $\phi_{3}^{(2)}\left(x^{\prime}\right), \phi_{4}^{(2)}\left(x^{\prime}\right)$, and $\phi_{5}^{(2)}\left(x^{\prime}\right)$ are completely arbitrary, and $x^{\prime}=\left(x_{2}, x_{3}, x_{4}, x_{5}\right)$. The system reduces to

$$
\dot{y}=A y+B v .
$$

Proof. The result follows directly by applying the result of Theorem 4.1 to (48) where $n=5, r=4, \mu=4, k_{1}=2$, and $k_{2}=k_{3}=k_{4}=1 ; p_{1}=2, \quad p_{2}=3, \quad p_{3}=4$, and $p_{4}=5$.

5.2. Permanent Magnet Synchronous Motor. The PM machine model [13] can be expressed as

$$
\dot{x}=A x+B u+f^{(2)}(x) ;
$$


$x=\left[x_{1}, x_{2}, x_{3}\right]^{t}=\left[i_{\mathrm{q} s}, i_{\mathrm{ds}}, \omega_{e}\right]^{t}$ and $u=\left[u_{1}, u_{2}\right]^{t}=\left[v_{\mathrm{qs}}, v_{\mathrm{ds}}\right]^{t}$ where $v_{\mathrm{qs}}, v_{\mathrm{ds}}, i_{\mathrm{qs}}$, and $i_{\mathrm{ds}}$ represent the quadrature and direct axes voltages and currents, respectively, and $\omega_{e}$ is stator angular velocity:

$$
\begin{gathered}
A=\left[\begin{array}{ccc}
-\frac{R_{s}}{L_{\mathrm{qs}}} & 0 & -\frac{I_{f}^{\prime} L_{\mathrm{dm}}}{L_{\mathrm{qs}}} \\
0 & -\frac{R_{s}}{L_{\mathrm{ds}}} & 0 \\
\frac{3 P^{2} L_{\mathrm{dm}} I_{f}^{\prime}}{8 J} & 0 & 0
\end{array}\right], \\
B=\left[\begin{array}{cc}
\frac{1}{L_{\mathrm{qs}}} & 0 \\
0 & \frac{1}{L_{\mathrm{ds}}} \\
0 & 0
\end{array}\right], \\
f^{(2)}(x)=\left[\begin{array}{c}
-\frac{L_{\mathrm{ds}}}{L_{\mathrm{qs}}} x_{2} x_{3} \\
\frac{L_{\mathrm{qs}}}{L_{\mathrm{ds}}} x_{1} x_{3} \\
\left(L_{\mathrm{ds}}-L_{\mathrm{qs}}\right) x_{1} x_{2}
\end{array}\right] .
\end{gathered}
$$

$I_{f}^{\prime}$ is the field current equivalent to the permanent magnet. $L_{\mathrm{ds}}, L_{\mathrm{qs}}$, and $L_{\mathrm{dm}}$ are the direct, quadrature, and magnetizing inductances, respectively. $R_{s}$ is the stator resistance. $P$ is the number of poles, and $J$ is the system moment of inertia. The model (55) can be reduced to the following normal form in a standard way using linear transformation of state variables [19] and linear state feedback, where $x, u, A, B$, and $f^{(2)}(x)$ are retained for simplicity of notation:

$$
\dot{x}=A x+B u+f^{(2)}(x),
$$

where $A$ and $B$ given by (2), where $r=\mu=2$ and $k_{1}=$ $2, k_{2}=1$, are

$$
\begin{gathered}
A=\left[\begin{array}{lll}
0 & 1 & 0 \\
0 & 0 & 0 \\
0 & 0 & 0
\end{array}\right], \quad B=\left[\begin{array}{ll}
0 & 0 \\
1 & 0 \\
0 & 1
\end{array}\right], \\
f^{(2)}(x)=\left[\begin{array}{l}
f_{1}^{(2)}(x) \\
f_{2}^{(2)}(x) \\
f_{3}^{(2)}(x)
\end{array}\right]=\left[\begin{array}{l}
C_{1} x_{2} x_{3} \\
C_{2} x_{3} x_{1} \\
C_{3} x_{2} x_{1}
\end{array}\right],
\end{gathered}
$$

with $C_{1}, C_{2}, C_{3}$ being constants.

Remark 5.3. $f^{(2)}(x)$ can be partitioned using (16) as

$$
f^{(2)}(x)=\left[\begin{array}{l}
f_{k_{1}}^{(2)}(x) \\
f_{k_{2}}^{(2)}(x)
\end{array}\right],
$$

where

$$
\begin{gathered}
f_{k_{1}}^{(2)}(x)=\left[\begin{array}{l}
f_{1}^{(2)}(x) \\
f_{2}^{(2)}(x)
\end{array}\right]=\left[\begin{array}{l}
C_{1} x_{2} x_{3} \\
C_{2} x_{3} x_{1}
\end{array}\right], \\
f_{k_{2}}^{(2)}(x)=\left[f_{3}^{(2)}(x)\right]=\left[C_{3} x_{2} x_{1}\right] .
\end{gathered}
$$

Note that $f_{1}^{(2)}(x)$ is a function of $x^{\prime}=\left(x_{2}, x_{3}\right)$ and hence $f_{1}^{(2)}(x)=f_{1}^{(2)}\left(x^{\prime}\right)$. The system given by (57) is in line with conditions of Theorem 4.1 as given in (30). Hence, Theorem 4.1 can be applied to system (57) to obtain the solution of generalized quadratic linearization which is given in Corollary 5.4.

Corollary 5.4. The system (57) can be quadratic linearized in the generalized sense using the transformation

$$
\begin{gathered}
y=x+\phi(x), \\
u=\left(I_{2}+\beta(x)\right) v+\alpha(x),
\end{gathered}
$$

where

$$
\begin{gathered}
\phi(x)=\phi^{(2)}(x)=\left[\begin{array}{c}
0 \\
f_{1}^{(2)}\left(x^{\prime}\right) \\
\phi_{3}^{(2)}\left(x^{\prime}\right)
\end{array}\right], \\
\alpha(x)=\alpha^{(2)}(x)=\left[\begin{array}{c}
-f_{2}^{(2)}(x) \\
-f_{3}^{(2)}(x)
\end{array}\right], \\
\beta^{(m-1)}(x)=(-1)^{m-1}\left\{B^{T} \frac{\partial \phi^{(2)}(x)}{\partial x} B\right\}^{m-1},
\end{gathered}
$$

where $\phi_{3}^{(2)}\left(x^{\prime}\right)$ is completely arbitrary and $x^{\prime}=\left(x_{2}, x_{3}\right)$. The system then reduces to

$$
\dot{y}=A y+B v .
$$

Proof. The result follows directly by applying the result of Theorem 4.1 to (57) where $n=3, r=2$, and $\mu=2 ; k_{1}=$ $2, k_{2}=1 ; p_{1}=2 ; p_{2}=3$.

5.3. Real-World Example of PMSM. The parameters of an actual machine are obtained from [20] as $R_{s}=1.2 \mathrm{ohms}$, $L_{\mathrm{qs}}=12.5 \mathrm{mH}, L_{\mathrm{ds}}=5.7 \mathrm{mH}, \omega_{e}=3500 \mathrm{rpm}, P=4, J=$ $0.0018 \mathrm{kgm}^{2}$, and $\lambda_{\mathrm{af}}=123 \mathrm{mWb}$ turns. The mathematical model of the machine in the normal form is given by

$$
\dot{x}=A x+B u+f^{(2)}(x),
$$

where $A$ and $B$ are as given in (57) and

$$
f^{(2)}(x)=\left[\begin{array}{l}
C_{1} x_{2} x_{3} \\
C_{2} x_{3} x_{1} \\
C_{3} x_{2} x_{1}
\end{array}\right]
$$


where $C_{1}=-0.165(e-3), C_{2}=-186.96$, and $C_{3}=$ 5.754386(e6). By applying Corollary 5.4 and choosing

$$
\begin{aligned}
\phi(x) & =\phi^{(2)}(x)=\left[\begin{array}{c}
0 \\
C_{1} x_{2} x_{3} \\
0
\end{array}\right]=\left[\begin{array}{c}
0 \\
-0.0165(e-3) x_{2} x_{3} \\
0
\end{array}\right], \\
\alpha(x) & =\alpha^{(2)}(x)=\left[\begin{array}{c}
\alpha_{1}^{(2)}(x) \\
\alpha_{2}^{(2)}(x)
\end{array}\right]=\left[\begin{array}{c}
186.96 x_{3} x_{1} \\
-5.754386(e 6) x_{2} x_{1}
\end{array}\right], \\
\beta^{(1)}(x) & =\left[\begin{array}{cc}
-C_{1} x_{3} & -C_{1} x_{2} \\
0 & 0
\end{array}\right] \\
& =\left[\begin{array}{cc}
0.0165(e-3) x_{3} & 0.0165(e-3) x_{2} \\
0 & 0
\end{array}\right], \\
\beta^{(m-1)}(x) & =(-1)^{m-1}\left\{B^{T} \frac{\partial \phi^{(2)}(x)}{\partial x} B\right\}^{m-1} .
\end{aligned}
$$

It is seen by verification that the system (64) becomes

$$
\dot{y}=A y+B v
$$

through transformation (61), given $\phi(x), \alpha(x)$, and $\beta(x)$ as above. The above system is completely linearized with no higher-order terms left behind.

5.4. Simulation Results. Verification of quadratic linearization of PMSM through simulations is given in an earlier paper by the authors [18]. The simulation results show that the closed-loop dynamic response of PMSM, before linearization, exhibits a behaviour that corresponds to varied responses for variations in reference and load conditions. However, it is verified that the quadratic linearized PMSM system gives a uniform closed-loop response for different reference and load conditions, as is expected of a linear system.

\section{Conclusion}

In this paper, linearization of machine models, which are predominantly quadratic in nature, is considered. Since the machine models only exhibit higher-order nonlinearities such as core loss, stray loss, and saturation, under extreme conditions, quadratic models can be used to represent the machines during normal operation.

The existing exact feedback linearization techniques introduce singularities in the system. This may result in the the faliure of linearization and the corresponding control technique, during the course of operation of the machine. Application of Poincare's approximate linearization technique due to Kang and Krener can remove the quadratic nonlinearity through a homogeneous transformation. This technique does not suffer from singularity issues but, being an approximate method, introduces higher-order terms in the process of quadratic linearization. Hence, in this paper, the concept of generalized quadratic linearization technique is introduced, wherein the higher-order terms introduced during the process of quadratic linearization are removed even as the quadratic term is removed.

A solution to the problem of generalized quadratic linearization is given for a class of control affine systems. Induction motor and PM motor models involving quadratic nonlinearity are considered and are quadratically linearised in the generalized sense. The proposed method can also be extended to wound rotor and synchronous machine models as well.

The generalized quadratic linearization technique proposed in this paper can also be considered as the approximate feedback linearization equivalent of exact linearization of a class of systems of the form (29). Since the solution of the generalized quadratic linearization involves removing thirdand higher-order nonlinearities, the result of the paper can also been seen as a contribution towards a special case of the open problem on arbitrary order linearization [21] of control affine systems.

Finally, as per the result of Theorem 4.1, $\beta(x)=$ $\sum_{m=2,3, \ldots} \beta^{m-1}(x)$ whose convergence needs to be established. Also, Theorem 4.1 of Section 4 deals only with sufficient conditions for generalized quadratic linearization. The question arises as to what are the necessary conditions for (9) to be generalized quadratically linearizable in general: this is our future work.

\section{References}

[1] G. S. Cardoso and L. Schnitman, "Analysis of exact linearization and approximate feedback linearization techniques," Mathematical Problems in Engineering Applications. In press.

[2] M. Bodson and J. Chiasson, "Differential-geometric methods for control of electric motors," International Journal of Robust and Nonlinear Control, vol. 8, no. 11, pp. 923-954, 1998.

[3] J. Chiasson, "Nonlinear controllers for an induction motor," Control Engineering Practice, vol. 4, no. 7, pp. 977-990, 1996.

[4] A. J. Krener, "Approximate linearization by state feedback and coordinate change," Systems and Control Letters, vol. 5, no. 3, pp. 181-185, 1984.

[5] V. I. Arnold, Geometric Methods in the Theory of Ordinary Differential Equations, Springer, New York, NY, USA, 1983.

[6] W. Kang and A. J. Krener, "Extended quadratic controller normal form and dynamic state feedback linearization of nonlinear systems," SIAM Journal on Control and Optimization, vol. 30, no. 6, pp. 1319-1337, 1992.

[7] A. J. Krener and W. Kang, "Extended normal forms of quadratic systems," in Proceedings of the 29th IEEE Conference on Decision and Control, pp. 2091-2096, December 1990.

[8] W. Kang, "Extended controller form and invariants of nonlinear control systems with a single input," Journal of Mathematical Systems, Estimation, and Control, vol. 4, pp. 253-256, 1994.

[9] I. A. Tall, "Feedback classification of multi-input nonlinear control systems," SIAM Journal on Control and Optimization, vol. 43, no. 6, pp. 2049-2070, 2000.

[10] I. A. Tall and W. Respondek, "Normal forms of two-inputs nonlinear control systems," in Proceedings of the 41st IEEE Conference on Decision and Control, pp. 2732-2737, 2002.

[11] R. Devanathan, "Linearization condition through state feedback," IEEE Transactions on Automatic Control, vol. 46, no. 8, pp. 1257-1260, 2001. 
[12] R. Devanathan, "Necessary and sufficient conditions for quadratic linearisation of a linearly controllable system," International Journal of Control, vol. 77, no. 7, pp. 613-621, 2004.

[13] B. K. Bose, Modern Power Electronics and AC Drives, Pearson Education,Inc., 2002.

[14] P. Tabuada and G. J. Pappas, "Finite Bisimulations of Controllable Linear Systems," in Proceedings of the 42nd IEEE Conference on Decision and Control, pp. 634-639, December 2003.

[15] P. Brunovsky, "A classification of linear controllable systems," Kybernetika, vol. 6, no. 3, pp. 173-188, 1970.

[16] B. A. Ogunnaike and W. H. Ray, Process Dynamics, Modeling and Control, Oxford University Press, 1994.

[17] A. K. Parvathy, V. Kamaraj, and R. Devanathan, "Application of quadratic linearization to the control of permanent magnet synchronous motor," in Proceedings of the 1st International Conference on Electrical Energy Systems, pp. 158-163, 2011.

[18] A. K. Parvathy, V. Kamaraj, and R. Devanathan, "'A generalized quadratic linearization technique for PMSM," European Journal of Scientific Research, vol. 54, no. 1, pp. 6-21, 2011.

[19] B. C. Kuo, Automatic Control Systems, Prentice-Hall, India, 2001.

[20] A. K. Parvathy and R. Devanathan, "Linearization of permanent magnet synchronous motor model," in Proceedings of the IEEE-ICIT Conference, pp. 483-487, 2006.

[21] R. Devanathan, "An arbitrary order linearization of linearly controllable systems," in 2002 MTNS Problem Book: Open Problems on the Mathematical Theory of Systems, V. D. Blondel and A. Megretski, Eds., Princeton University Press, 2003. 

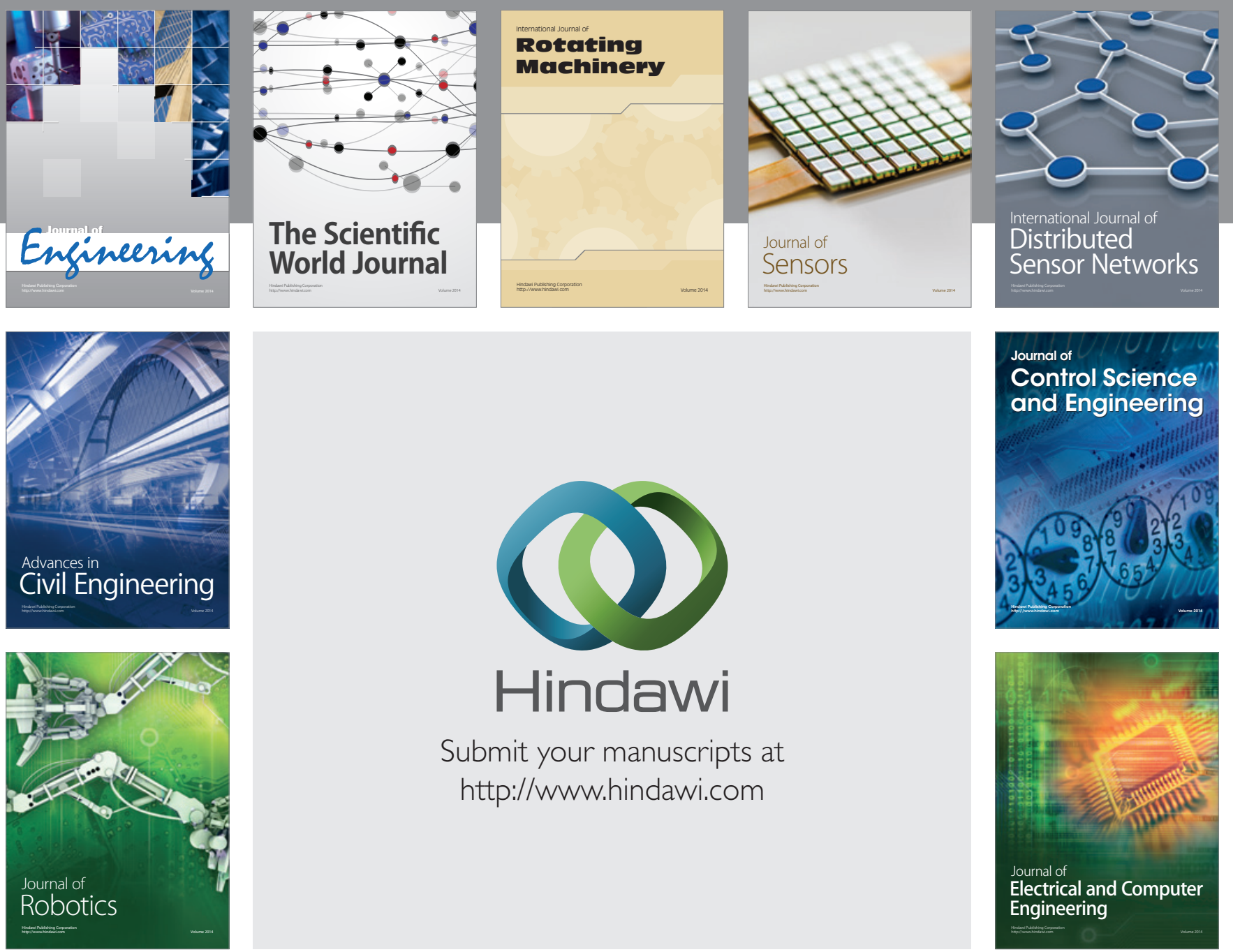

Submit your manuscripts at

http://www.hindawi.com
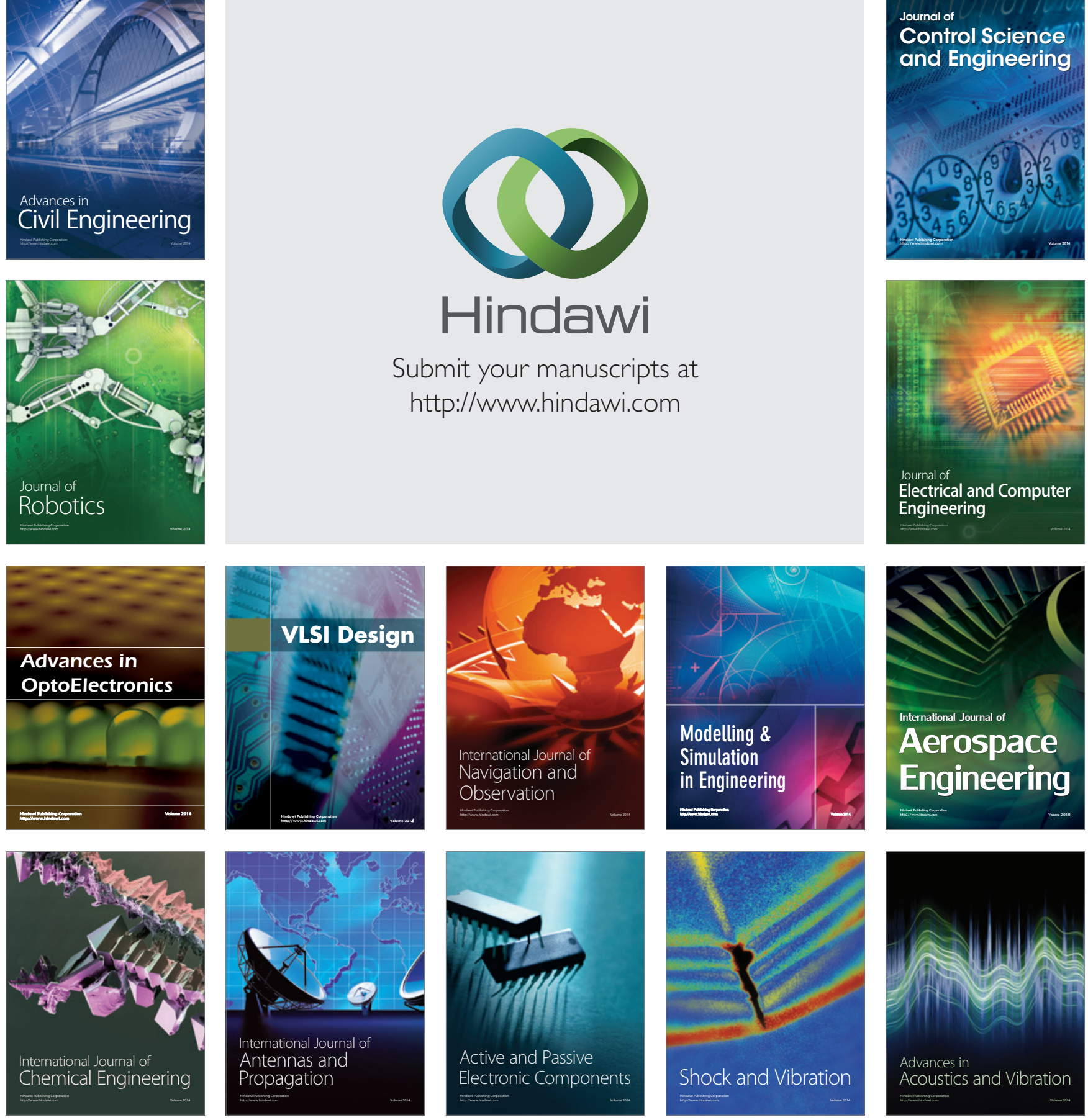